\title{
Cast-as-Intended Mechanism with Return Codes Based on PETs Extended Version
}

\author{
Achim Brelle and Tomasz Truderung \\ Polyas $\mathrm{GmbH}$ \\ a.brelle@polyas.de, t.truderung@polyas.de
}

\begin{abstract}
We propose a method providing cast-as-intended verifiability for remote electronic voting. The method is based on plaintext equivalence tests (PETs), used to match the cast ballots against the pregenerated encrypted code tables.

Our solution provides an attractive balance of security and functional properties. It is based on well-known cryptographic building blocks and relies on standard cryptographic assumptions, which allows for relatively simple security proofs. Our scheme is designed with a built-in fine-grained distributed trust mechanism based on threshold decryption. It, finally, imposes only very little additional computational burden on the voting platform, which is especially important when voters use devices of restricted computational power such as mobile phones. At the same time, the computational cost on the server side is very reasonable and scales well with the increasing ballot size.
\end{abstract}

\section{Introduction}

Modern electronic voting systems are expected to provide a combination of security guarantees which includes, most importantly, ballot secrecy and end-to-end verifiability. For the latter, one crucial part is so-called cast-as-intended verifiability which means that a voter has means to make sure that the ballot cast on his or her behalf by the voting client application and recorded by the voting server contains the intended voting option, as chosen by the voter. This property must be guaranteed without assuming that the voter platform is honest. Indeed, such assumption would be unjustified especially in the context of remote voting, where voting client programs (typically HTML/JS applications) run on voters' devices. One cannot reasonably assume that such devices are properly maintained, patched and free of malware. Moreover, as often the code of the voting client application is served by the voting server, such trust assumption would have to be extended to such servers as well.

The problem of providing adequate and usable solutions for cast-as-intended verifiability has recently attracted significant attention. In particular, various solutions based on the idea of return codes have been proposed [2, 7], 13, 
[8, [5], 910, where different solutions provide different balance of security and usability features. Notably, solutions based on return codes [7/8/15] were used in Norway in legally binding municipal and county council elections in 2011 and 2013, [5] was used in 2015 in binding elections in the Swiss canton of Neuchâtel, while [9], as stated in the paper, is planned to be used as a part of the electronic voting system for the State of Geneva (Switzerland) [10.

The above mentioned solutions share the following underlying idea. In the registration phase, each voter obtains over a trusted channel a ballot sheet, where pre-generated return codes (typically short random alpha-numeric sequences) are printed next to each voting choice. Then, in the voting phase, after the voter has selected her voting choices and the voting client application has submitted an encrypted vote to the remote voting server, the voting authorities compute/retrieve (in some way dependent on the specific solution) return codes which are meant to correspond to the choices made by the voter. These codes are sent back to the voter (possibly using an independent channel) who compares them with the codes printed on her ballot sheet next to the selected choices. The idea here is that when this match succeeds, the voter can be sure that the submitted (encrypted) vote indeed contains her intended choices (as otherwise the voter would not have obtained the matching codes). The voter may then finalize the ballot casting process (by, for instance, submitting some kind of finalization code) or, if the match does not succeed, she may undertake different steps (for instance, vote from another device or use a completely different voting method).

Our Contribution. In this paper we propose a new cast-as-intended mechanism based on return codes. Our solution provides an attractive balance of security and functional properties:

1. It is based on well-known cryptographic building blocks and relies on standard cryptographic assumptions, which allows for relatively simple security proofs. In fact, our proofs are modular in that they do not depend on the details of the underlying voting protocol to which our return code scheme is added. In particular, our formal security results hold for systems obtained by adding our return codes to the mix-net-based variants of the well-known Helios and Belenios protocols.

2. Our scheme is designed with distributed trust in mind: the computations carried out to retrieve/compute return codes are distributed in their nature, such that a threshold of trustees must be corrupted in order to carry out a successful attack and fool the voter.

3. Our solution imposes only very little additional computational burden on the voting platform, which is especially important if voters use devices of restricted computational power such as mobile phones. The computational cost on the server side is very reasonable and scales well with the increasing ballot size (it is, up to ballots of fairly big size, essentially constant).

Our scheme is meant to provide cast as intended verifiability even if the voting platform is controlled by the adversary under the following assumptions. 
First, we assume that not more than $t-1$ tellers are corrupted (i.e. controlled by the adversary), where $t$ is the threshold of the used threshold-decryption scheme. Second, we assume that the printing facility and the ballot delivery channel are not corrupted. Under these assumptions, if the voter during the voting process obtains the expected return codes (that is the codes printed on her ballot sheet next to her intended choices), then the cast ballot is guaranteed to contain the intended voter's choice.

We note that the second assumption is shared with other return code solutions. It is a strong assumption and requires special measures in order to be justified in specific deployments. The same assumption (in addition to the standard assumptions that the voter platform is honest and that at most $t-1$ tellers are corrupted) is necessary for voters' privacy. Finally, note that our scheme (similarly to most of the return code solutions; see below for more discussion) is not meant to provide receipt freeness.

On the technical level, our scheme is inspired by the PGD system [1411] which however does not implement the idea of returns codes, but instead the one of voting codes (where a voter submits codes corresponding to her choice). Sharing some similarities with this construction, our system differs substantially from PGD in many aspects.

As an additional contribution of this paper, we demonstrate an attack on a return code scheme recently proposed in $9[10$ which was planned to be used in the context of the Geneva Internet voting project (see below for more details).

Related Work. As already mentioned, our scheme is inspired by the PGD system 1411 and, on the technical level, uses some similar ideas: it uses distributed PETs (plaintext equivalence tests) to match the submitted ballots against a pre-published encrypted code table. Our scheme, however, differs from PGD in some significant ways. Our scheme scales better with increasing ballot complexity (PGP performs one PET for every entry in the voter's code table; we perform only one PET per voter even for relatively complex ballots). On the technical level we avoid the use of encrypted permutations (onions). Finally, PGD uses the idea of voting codes, where a voter submits codes corresponding to the chosen candidates (although the authors also suggest the possibility of using return codes). We note here that the use of voting codes (as in PGD) results in stronger ballot secrecy (the voting client does not get to learn how the voter's choice and hence it does not have to be trusted for ballot secrecy). As a trade-off, using voting codes tends to be less convinient for the voters.

In a series of results including [213/7/8, related to the Norwegian Internet voting projects (eValg2011 and eValg2013) [15, the underlying, shared idea is as follows. The code for a voting option $v$ (which is cast in an encrypted form $\operatorname{Enc}_{p k}(v)$ ) is deterministically derived from $v$ using a per-voter secret $s$ (it typically is $v^{s}$ ). This derivation process is carried out by two servers (playing fixed, specific roles) in such a way that if only one of them is corrupted, the security goal of the return codes is not subverted. In order to make this idea work for 
more complex ballots, 7/8 uses a technique of combining codes, which however requires some non-standard cryptographic assumption (hardness of the SGSP problem, where SGSP stands for Subgroup Generated by Small Primes). These schemes (as opposed to ours) do not allow for more fine-grained distribution of trust: there are exactly two parties with specific roles, one of which must be honest.

The above idea was further transformed in a scheme proposed for the voting system in the canton of Neuchâtel in Switzerland [5], with the main technical difference that in this system a voter holds one part of the secret used for code generation (which causes some usability issues which were addressed by introducing of a so-called usability layer, which unfortunately weakens security guarantees). Security of this construction relies on the same non-standard security assumption as 78 do and, similarly, there is no built-in fine grained mechanism for distributed trust. Compared to our system, this system requires much more complex computations on the voting platform, but less computations for the election authorities (although in both cases the ballot processing time on the server side is essentially constant independently of the number of voting options).

Recently, an interesting solution has been proposed in the context of the Geneva Internet voting project [9]10. This solution is based on oblivious transfer, where, intuitively, the security of the mechanism is provided by the fact that the authorities (even although they may know all the codes) do not know which codes are actually transfered to the voter. This provides some level of protection against vote buying schemes which otherwise could be very easily mounted by a dishonest authority (if a voter was willing to disclose her ballot sheet). To our knowledge, this is the only return-codes scheme with this property.

As a downside, in this protocol codes cannot be transfered using an independent channel (they must be transfered via the voter's platform), which rules out the use of this protocol in elections where re-voting is allowed. Furthermore, this protocol, again, uses the same non-standard cryptographic assumption as 788 .

Finally, as already mentioned, we have discovered a serious flaw in this construction, described in detail in Appendix B. Our attack violates the cast-asintended property of the scheme (the voter cannot be sure that the cast ballot represents her intended choice even if she receives the expected return codes) and can be mounted by an attacker who only controls the voting platrofm. In short, we show that such an attacker (which is exactly the kind of attacker the system is meant to defend against) can cast invalid ballots and still provide the voters with valid return codes. These invalid ballots are accepted by the voting server, tallied, and only discovered and rejected after tallying, when the link between the ballot and the voter has been hidden. Note that even if augmented the protocol with a mechanism enabling us to trace the mallformed decrypted ballots back to the voters, it would only point to dishonest voters' devices which cannot be held accuntable.

While there is a natural countermeasure for this attack (adding appropriate zero-knowledge proofs of well-formedness of the ballot), it comes with significant 
degradation of performance: it works, roughly, in quadratic time with respect to the number of voting options, which renders this solution impractical for bigger ballots 1

Structure of the paper. After introducing some preliminary definitions in (Section 2) and providing an overview of the election process (Section 3), we describe in Section 4 a simple variant our scheme, applicable only for ballots with one binary choice. The general variant is described in Section 5 after which the security analysis is presented in Section 6. In appendices we provide some further details and demonstrate the mentioned attack on 9 .

\section{Preliminaries}

Our return code scheme uses the well-known ElGamal cryptosystem over a cyclic group $G$ of quadratic residues modulo a safe prime $p=2 q+1$. This cryptosystem is multiplicatively homomorphic (that is $\operatorname{Enc}_{p k}(m) \cdot \operatorname{Enc}_{p k}\left(m^{\prime}\right)$ results in an encryption $\operatorname{Enc}_{p k}\left(m \cdot m^{\prime}\right)$ if $m$ and $m^{\prime}$ are elements of the underlying group). A distributed key generation protocol for the ElGamal cryptosystem (where $n$ tellers jointly generate a secret key and the corresponding public key, and pre-determined threshold $t<n$ out of $n$ tellers is necessary for decryption) is proposed, for instance, in [6].

A plaintext-equivalence test [12] is a zero-knowledge protocol that allows the (threshold of) tellers to verifiably check if two ciphertexts $c$ and $c^{\prime}$ contain the same plaintext, i.e. to check if $\operatorname{Dec}_{s k}(c)=\operatorname{Dec}_{s k}\left(c^{\prime}\right)$, but nothing more about the plaintexts of $c$ and $c^{\prime}$.

Our return codes solution can be added to any voting system with encrypted ballot of a form which is compatible with our scheme in the following sense: (1) ElGamal cryptosystem with threshold decryption, as introduced above, is used to encrypt voters' choices and (2) ballots contain zero-knowledge proofs of knowledge of the encrypted choices (which is a very common case); additionally, for the general case, we require that (3) voters' choices are encoded in a specific way (see Section 5) before encryption. We do not fix details of the authentication mechanism nor those of the tallying process. In fact, our security proofs work independently of these details. Examples of voting systems compatible with our scheme are Helios [1] and Belenios [4] with mix-net-based tallying and, for the simple variant, also with homomorphic tallying.

\footnotetext{
${ }^{1}$ We contacted the authors who confirmed the flaw and are working on a countermeasure for the attack. According to the authors (personal communication, May 15, 2017), they were able to find a solution which avoids quadratic time computations during the ballot casting, assuming instead quadratic-time pre-computations carried out by servers in the off-line phase.
} 


\section{Overview of the Election Process}

In this section we present an overview of the voting process. Because our scheme (like other return codes solutions) is aimed at providing cast-as-intended verifiability even when the voting platform is potentially corrupted, we make the distinction between voters and their voting platform, that is devices, including the software potentially served by the voting server, voters use to cast ballots.

The election process is run by the set of authorities including:

- Tellers who jointly generate the public election key $p k_{e}$ key and share the corresponding decryption key in a threshold manner. They also, similarly, jointly generate the public code key $p k_{c}$ which will be used to encrypt codes in code tables and an auxiliary public key $p k_{a}$ for which the corresponding secret key is known to every teller (here we do not need threshold decryption and use any CCA2-secure cryptosystem). The tellers take part in code table generation and generation of additional codes for voters (authentication, finalisation and confirmation codes). They may also carry out additional steps (such as ballots shuffling), as specified by the underlying protocol.

- Secure bulletin boards which, traditionally for e-voting systems, are used by voting authorities to publish results of various steps of the election procedure, including the final election result. Secure bulletin boards provide appendonly storage, where records can be published (appended) but never changed or removed.

- Voting server which is responsible for voters' authentication and ballot recording (where a ballot is published on a designated secure bulletin board).

- Printing facility, including the ballot sheets delivery, used to print ballot sheets in a trusted way and to deliver ballot sheets to eligible voters. The printing facility, in the setup phase generates its private/public encryption key pair and publishes the public key $p k_{p}$.

Our return code schemes supports the following, general ballot structure: a ballot may contain a number of voting options (candidates), where a voter can independently select each of these options (or, put differently, provide 'yes'/'no' choice independently for each voting option). Further restrictions can be imposed (such as for example, that exactly $k$ or at most $k$ options are selected) and checked after the ballots are decrypted. Note that with this ballot structure we can encode different types of ballots, such as for instance, ballots where each candidate can get more than one vote.

The election process consists of the following voting phases:

In the setup phase the tellers and the printing facility generate keys and codes, as described above. In the registration phase every eligible voter obtains (via a trusted channel) a ballot sheet. The ballot sheet contains an authentication code (used as a authentication measure; we abstract here from the details of the authentication mechanism and simply assume that a mechanism with sufficient security level is used), a finalization code, a confirmation code, and a list of voting 
options (candidates) with printed next to each of them two return codes: one for the 'no' choice and one for the 'yes' choice.

In the voting phase, the voter, using her voting platform and the authentication code, authenticates to the voting server and selects her choices. The voting platform creates a ballot with the selected choices and submits it to the voting server. The ballot is then processed by the voting authorities who send back to the voter (via the voting platform or via some other, independent channel) sequence of return codes that correspond to the cast (encrypted) choices. The voter compares the obtained codes with the ones printed on her ballot sheet to make sure that they indeed correspond to her intended choices. If this is the case, the voter provides the voting platform with the finalization code which is forwarded to the voting server. Given this finalization code, the voting server sends the confirmation code to the voter and completes the ballot casting process by adding the ballot to the ballot box. If something does not work as expected (the voter does not get the expected return codes or does not obtain the confirmation code after providing her finalisation code), the voter can undertake special steps, as prescribed by the election procedure (use, for instance, another device or the conventional voting method).

Finally, in the tallying phase, the ballots published on the ballot box are tallied and the result is computed.

\section{The Variant with one Binary Choice}

In this section, we present a simple variant of our scheme, where the ballot contains only one binary choice (two candidate races or 'yes'/'no' elections). This variant, while avoiding the technical details of the general variant, demonstrates the main ideas of the scheme.

Code table and ballot sheet. As shortly mentioned before, in the setup phase, the voting authorities generate for every voter an encrypted code table. We will now only describe the expected result of the code generation procedure, without going into the detail. Such details will be given in Section 5.2, where the general case is covered (which subsumes the simple case discussed in this section). We only mention here that code tables are generated in fully verifiable way.

The code generation procedure generates, for every voter, two random codes $c_{0}$ and $c_{1}$, corresponding to the 'no' and 'yes' choice, and a random bit $b$, called a flip bit. It also generates for every voter a random finalization code and a confirmation code. Additionally, we assume that some kind of authentication codes for voters may be generated by this procedure as well, but we abstract away from the details of the authentication mechanism, as the presented construction does not depend on them.

The ballot sheet (delivered to the voter over a trusted channel) contains the authentication, finalization, and confirmation codes, the return codes $c_{0}$ and $c_{1}$ printed in clear next to, respectively, the 'no' and the 'yes' voting choice, 
and the flip bit $b$. For usability reasons, the flip bit can be integrated into the authentication code, so that the voter does not have to enter it separately.

The code table associated with the voter, published on a bulletin board, is of the form

$$
c_{\text {fin }}, e_{\text {conf }},\left(e_{0}, d_{0}\right),\left(e_{1}, d_{1}\right)
$$

where $c_{\text {fin }}$ is a commitment to the finalization code, $e_{\text {conf }}$ is encryption of the confirmation code under $p k_{c}$ and

$$
e_{0}=\operatorname{Enc}_{p k_{e}}(b), \quad d_{0}=\operatorname{Enc}_{p k_{c}}\left(c_{b}\right), \quad e_{1}=\operatorname{Enc}_{p k_{e}}(1-b), \quad d_{0}=\operatorname{Enc}_{p k_{c}}\left(c_{1-b}\right) .
$$

Note that the this record contains the pair of ciphertexts corresponding to the 'no' choice (encrypted 0 and encrypted code $c_{0}$ ) and the pair of ciphertexts corresponding to the 'yes' choice (encrypted 1 and encrypted code $c_{1}$ ). The order in which these two pairs are placed depends on the flip bit (if the flip bit is 1 the order is flipped) 2

Ballot casting. The voter provides her voting application with her authentication code, the flip bit $b$, and her voting choice $v \in\{0,1\}$. The voting application produces a ballot containing

$$
w=\operatorname{Enc}_{p k_{e}}(v), \operatorname{Enc}_{p k_{a}}(\tilde{b}), \pi
$$

where $\tilde{b}=v \oplus b$ and $\pi$ is a zero-knowledge proof of knowledge of the plaintext in the ciphertext $w(\tilde{b}$ is encrypted in order to hide it from an external observer; the tellers will decrypt this value in the next step).

The voting authorities check the zero-knowledge proof $\pi$, decrypt $\tilde{b}$, select $e_{\tilde{b}}$ from the voter's table and perform the PET of this ciphertext with the ciphertext $w$ submitted by the voter's platform. It is expected that this PET succeeds (which is the case if the voting platform follows the protocol and the ballot sheet and the code table are correctly generated). If this is the case, the corresponding encrypted code $d_{\tilde{b}}$ is decrypted (which should result in $c_{v}$ ) and delivered to the voter. The voter makes sure that, indeed, the return code is $c_{v}$, i.e. it corresponds to the voting choice $v$, before she provides her finalization code (in order to finalize the ballot casting process). The voting authorities check that the provided finalization code is a valid opening for the commitment $c_{\text {fin }}$. If this is the case, they finalise the ballot casting process: they jointly decrypt the confirmation code, send it to the voter, and add the voter's ballot to the ballot box.

Tallying. Finally, after the voting phase is over, ballots collected in the ballot box are tallied. We abstract here from the details of the tallying procedure. Importantly, our security results work regardless of the details of this procedure.

\footnotetext{
${ }^{2}$ Note that the plaintext are first mapped into $G$ before being encrypted; for an appropriate choice of the mapping, we obtain a system which coincides with the general variant with $k=1$ and, furthermore, allows for homomorphic tallying.
} 
The intuition behind security of this scheme is as follows. Because, of the correctness of the code table and PET operations (which is ensured by zero-knowledge proofs), if the PET succeeds, then the decrypted code must be the return code corresponding to the actual plaintext in the encrypted ballot. To fool the voter, an adversary would have to send him the code contained in the second ciphertext which has not been decrypted. But the best the adversary can do - not being able to break the used encryption scheme - is blindly guess this code, which gives him very small probability of success.

Remark 1. For this simple variant, we do not really need to include the flip bit in the ballot sheet: the ciphertext $w$ could be matched, using the PET protocol, against both $e_{0}$ and $e_{1}$, one of which should succeed, which would determine $\tilde{b}$. Including the flip bits in the ballot sheets is however crucial for efficiency of the general variant.

We can note that the additional computational cost of this scheme added to the voting platform is only one encryption. The computational cost incurred by this scheme on the server side (per one voter) is one additional decryption to decrypt $\tilde{b}$, one verifiable PET, and one distributed decryption to decrypt the return code.

As we will see in a moment, the general variant of our scheme (with $k$ independent choices) can be seen as a combination of $k$ simple cases as described here with some optimisations. Interestingly, with these optimisations, the additional computational cost incurred by our scheme - if the size of the ballot does not grow too much - remains essentially the same.

\section{The General Variant}

In this section we present the general variant of our code voting scheme, where ballots can contain some number $k$ of independent binary choices, one for each voting option. This variant is expressive enough to handle wide variety of complex ballots. Despite some technical details used for optimisation, this variant shares the same underlying idea, illustrated by the simple variant.

We assume some encoding $\gamma$ of the voting options $1, \ldots, k$ as elements of the group $G$ such that the voter's choice, which is now a subset of individual voting options, can be encoded as the multiplication of the encodings of these individual options. Of course, we assume that the individual voting options can be later efficiently retrieved from such an encoding. As an example of such encoding we can use the technique used for instance in [59], where the voting options are encodes as small prime numbers which belong to the group $G$.

Similarly, we assume a family of efficient encodings $\delta_{i}(i \in\{1, \ldots, k\})$ from the set of return codes to the group $G$, such that individual codes $c_{1}, \ldots, c_{k}$ can be efficiently extracted from the product $\delta_{1}\left(c_{1}\right) \cdots \delta_{k}\left(c_{k}\right)$. An example of such an encoding is given in Section $\mathrm{A}$. 


\subsection{Ballot Structure and Voting Procedure}

Code table and ballot sheets. The code generation procedure is described in details in Section 5.2 In addition to finalisation and confirmation codes which are generated as previously, this procedure generates, for every voter and every voting option $i \in\{1, \ldots, k\}$, two random codes $c_{i}^{0}$ and $c_{i}^{1}$ corresponding to, respectively, the 'no' and 'yes' choice. It then generates a random sequence of flip bits $\boldsymbol{b}=b_{1}, \ldots, b_{k}$, where $b_{i} \in\{0,1\}$.

The ballot sheet sent to the voter contains now, besides the authentication, finalisation, and confirmation codes, return codes $\left(c_{1}^{0}, c_{1}^{1}\right), \ldots,\left(c_{k}^{0}, c_{k}^{1}\right)$ printed in clear next to corresponding voting options and marked as, respectively the 'no' and the 'yes' choice. It also contains the flip bits $\boldsymbol{b}$ (as before, this vector can be integrated in the authentication code).

The published code table associated with the voter contains, as before $c_{\text {fin }}$, $e_{\text {conf }}$ and

$$
\left(u_{i}^{0}, u_{i}^{1}\right)_{i=0}^{k}=\left(t_{i}^{b_{i}}, t_{i}^{1-b_{i}}\right)_{i=0}^{k}
$$

where

$$
t_{i}^{0}=\left(\operatorname{Enc}_{p k_{e}}(1), \operatorname{Enc}_{p k_{c}}\left(\delta_{i}\left(c_{i}^{0}\right)\right) \quad \text { and } \quad t_{i}^{1}=\left(\operatorname{Enc}_{p k_{e}}(\gamma(i)), \operatorname{Enc}_{p k_{c}}\left(\delta_{i}\left(c_{i}^{1}\right)\right) .\right.\right.
$$

Note that $t_{i}^{0}$ corresponds to the 'no' choice (it contains an encryption of 1 and the encoded code for 'no') and $t_{i}^{1}$ corresponds to the 'yes' choice (it contains an encryption of the encoded option $i$ and the encoded code for 'yes'). Note also that $u_{i}^{b_{i}}=t_{i}^{0}$ and $u_{i}^{1-b_{i}}=t_{i}^{1}$.

Ballot casting. The voter provides her voting application with her voting choice $v_{1}, \ldots, v_{k} \in\{0,1\}$ and the bit sequence $\boldsymbol{b}$. The voting application computes $v=\prod_{i \in V} \gamma(i)$, where we define $V$ as the set $\left\{j: 1 \leq j \leq k, v_{j}=1\right\}$, and produces a ballot containing

$$
w=\operatorname{Enc}_{p k_{e}}(v), \operatorname{Enc}_{p k_{a}}(\tilde{\boldsymbol{b}}), \pi
$$

where $\pi$ is, as before, a zero-knowledge proof of knowledge of the plaintext of $w$ and $\tilde{\boldsymbol{b}}=\tilde{b}_{1}, \ldots, \tilde{b}_{k}$ with $\tilde{b}_{i}=b_{i} \oplus v_{i}$.

The voting authorities decrypt $\tilde{\boldsymbol{b}}$ and select the values $w_{i}=u_{i}^{\tilde{b}_{i}}$, for $i \in$ $\{1, \ldots, k\}$. Note that if the voter has not chosen the $i$-th election option, then $w_{i}=u_{i}^{b_{i}}=t_{i}^{0}$, by the definition of $u$. Otherwise, $w_{i}=u_{i}^{1-b_{i}}=t_{i}^{1}$.

The voting authorities multiply $w_{1}, \ldots, w_{k}$ (component-wise) obtaining the pair $\left(e^{*}, c^{*}\right)$, where $e^{*}$ should be (if the voter platform followed the protocol) encryption of $v=\prod_{i \in V} \gamma(i)$. The voting authorities perform the PET of $e^{*}$ with the encrypted choice $w$ from the ballot. If this PET fails, the casting procedure is canceled. Otherwise, the decryption tellers jointly decrypt $c^{*}$. Observe that, by the properties of the published code table, this decrypted value is the product of $\delta_{j}\left(c_{j}^{v_{j}}\right)$, i.e. it is the product of the codes corresponding to the choices made by the voter. This value is decomposed into individual codes $c_{1}^{v_{1}}, \ldots c_{k}^{v_{k}}$ and sent 
to the voter (via the voting platform or an independent channel). As before, the voter makes sure that the received codes correspond to her choices before providing the finalisation code.

Note that the ballot processing on the server side only requires one verifiable PET, one decryption and one threshold decryption, independently of the number $k$ of the voting options, plus some number of multiplications and divisions (which depends on $k$ ), as long as $k$ codes can be efficiently represented as one element of the group $G$ which is in detail discussed in Section $\mathrm{A}$

\subsection{Code Table Generation}

The code table generation presented below is fully verifiable. Note that we could also consider a version without zero-knowledge proofs, but with partial checking instead, where a bigger number of records is produced and the some of them (randomly selected) are open for audit.

We will assume that the code generation procedure is carried out by the tellers, but it can by carried out by any set of independent parties, as it does not require possession of any secret keys. We will present here a version, where, for the same voting option, distinct voters obtain distinct codes, although different variants are also possible (and may be useful if the number of voters is very big).

The set of codes is Codes $=\{1, \ldots, m\}$ with $m>2 n$, where $n$ is the number of voters (reasonable values for $m$, that is values corresponding to desired security levels, can be determined using Theorem 3).

For simplicity of presentation, in the following, we will leave out handling of the authentication, finalization and confirmation codes. The procedure consists of the following steps.

1. For every voting option $j$, the tellers deterministically compute

$$
\operatorname{Enc}_{p k_{c}}\left(\delta_{j}(1)\right), \operatorname{Enc}_{p k_{p}}(1), \ldots, \operatorname{Enc}_{p k_{c}}\left(\delta_{j}(m)\right), \operatorname{Enc}_{p k_{p}}(m) .
$$

where all the ciphertext are obtained using the pre-agreed randomness 1 .

2. The tellers shuffle the above sequence of ciphertexts using a verifiable mix net obtaining a sequence of the form

$$
\operatorname{Enc}_{p k_{c}}\left(\delta_{j}\left(c_{1}\right)\right), \operatorname{Enc}_{p k_{p}}\left(c_{1}\right), \ldots, \operatorname{Enc}_{p k_{c}}\left(\delta_{j}\left(c_{m}\right)\right), \operatorname{Enc}_{p k_{p}}\left(c_{m}\right),
$$

where $c_{i}=\pi(i)$ for some permutation $\pi$ and the ciphertext are re-randomized. Note that for this we need to use a version of verifiable mixing which applies the same permutation (but independent re-randomization factors) to pairs of ciphertexts. Such generalizations of know verifiable shuffling algorithms are possible 3

\footnotetext{
${ }^{3}$ In particular, it is straightforward to generalize the shuffle protocol of $[3]$ to provide such functionality.
} 
3. The tellers take the consecutive encrypted codes produced in the previous step and organize them into the records of the following form, one for each voter $i$ :

$$
\begin{aligned}
\left\{\operatorname{Enc}_{p k_{p}}(0), \operatorname{Enc}_{p k_{p}}\left(c_{j}^{\prime}\right), \operatorname{Enc}_{p k_{p}}(1), \operatorname{Enc}_{p k_{p}}\left(c_{j}^{\prime \prime}\right),\right. \\
\left.\quad \operatorname{Enc}_{p k_{e}}(1), \operatorname{Enc}_{p k_{c}}\left(\delta_{j}\left(c_{j}^{\prime}\right)\right), \operatorname{Enc}_{p k_{e}}(\gamma(j)), \operatorname{Enc}_{p k_{c}}\left(\delta_{j}\left(c_{j}^{\prime \prime}\right)\right)\right\}_{j \in\{1, \ldots, k\}}
\end{aligned}
$$

where the ciphertext with (encoded) choices are generated deterministically with the randomness 1 .

4. The tellers perform, one after another, series of micro-mixes for every such a record: Each teller, for the input record $R=\left(a_{1}, b_{1}, a_{2}, b_{2}, a_{1}^{\prime}, b_{1}^{\prime}, a_{2}^{\prime}, b_{2}^{\prime}\right)$ (which is the output of the previous teller or, for the first teller, the record produced in the previous step) picks a random bit. If this bit is 0 , then it only re-encrypts all the elements $R$. If the flip bit is 1 , then, in addition, it accordingly flips the elements of the record and outputs a re-encryption of $R^{\prime}=\left(a_{2}, b_{2}, a_{1}, b_{1}, a_{2}^{\prime}, b_{2}^{\prime}, a_{1}^{\prime}, b_{1}^{\prime}\right)$. The teller produces a zero-knowledge proof of correctness of this operation (such step can be implemented as a verifiable mixing operation; it can be also realized using disjunctive ChaumPedersen zero-knowledge proofs of the fact that the resulting record is either a re-encryption of $R$ or $R^{\prime}$ ).

5. The parts of the records encrypted with $p k_{c}$ and $p k_{e}$ are published in voters' code tables. The parts encrypted with $p k_{p}$ are given to the printing facility which decrypts the records. The decrypted content contains the return codes and (implicitly, via the order of plaintexts) the flip bit sequence $\boldsymbol{b}$.

Because, in the above procedure, all the operation are fully deterministic or with appropriate zero-knowledge proofs, we obtain the following result.

Theorem 1. The above procedure is corrects, i.e. it produces correctly linked ballot sheets and encrypted code tables with overwhelming probability. Moreover, unless the threshold of trustees are dishonest, only the printing facility learns how codes are distributed amongst voters.

\section{Security Analysis}

As noted in the introduction, coercion resistance and receipt-freeness are not the goals of our scheme. In fact, the use of return codes, as in many similar solutions, specifically makes the scheme prone to vote selling if dishonest authorities are involved in the malicious behaviour.

The results presented in this section are stated for the case where re-voting is not allowed. For the case with re-voting (casting multiple ballots, of which, say, the last is counted), we expect that the privacy result holds (we leave however the proof for future work), while only a weaker form of cast-as-intended verifiability than the one presented in Section 6.2 can be guaranteed: namely, we have to assume that an independent channel is used to send return codes to voters and that both the tellers (who see the sent return codes) and this channel are honest. 


\subsection{Ballot Secrecy}

Ballot secrecy means, informally, that it is impossible (for an adversary) to obtain more information about the choices of individual honest voters (that is voters following the protocol), than can be inferred from the explicit election result. Our code voting scheme provides voters privacy under the following assumptions:

P1. The voting platform is not corrupted.

P2. At most $t-1$ tellers are corrupted, where $t$ is the threshold for decryption. P3. The printing facility and the ballot sheet delivery channel are not corrupted.

The first two assumptions are standard and for voters' privacy and shared by many e-voting protocols (using and not using return codes). The third assumption is also shared by any code voting scheme (where codes need to be printed and delivered to the voter). Therefore, in this sense, these are the minimal assumptions for electronic voting with return codes.

Note also, that the informal definition of privacy given above only protect honest voters who, in particular, do not reveal their ballot sheet to another parties, excluding voters who want to sell their ballots.

We formalize the above notion of privacy using the following game between the adversary and the system $P$ representing the honest components of the evoting system, where the adversary gets to control all but two (honest) voters. For simplicity of presentation, we consider here the simple case where voters have only one yes/no choice. We will consider two variants of $P$ : variant $P_{0}$, where the first of the honest voters votes for the 'no' option and the second honest voters chooses the 'yes' option, and variant $P_{1}$, where the choices of the honest voters are swapped. With these definitions, we express the notion of privacy by requiring that there is no polynomially bounded adversary $A$ which can detect if he is interacting with $P_{0}$ or $P_{1}$ (up to some negligible probability), that is:

$$
\operatorname{Prob}\left[P_{0} \| A \mapsto 1\right] \equiv_{\text {negl }} \operatorname{Prob}\left[P_{1} \| A \mapsto 1\right]
$$

where $P_{i} \| A \mapsto 1$ denotes the event that in the run of the system composed of $P_{i}$ and $A$, the adversary outputs 1 (accepts the run). We assume that the adversary can interact with the system in the following way: it interacts with the honest tellers playing the role of the dishonest tellers (in all the protocol stages). It also casts ballots of the dishonest voters and, at some chosen time, triggers the honest voters to cast their ballots.

We will now establish our privacy result in a modular way, independently of many details of the underlying voting system to which our return code scheme is added. We only assume that the system has the structure which allows for the described above game and which uses ballot encoding 'compatible' with our construction, as described in Section 2 .

Theorem 2. Let $U$ be the underlying voting protocol and let $P$ denote the protocol obtained from $U$ by adding our return code scheme. If $U$ provides ballot secrecy, as expressed by (10), then $P$ provides secrecy as well. 
In the proof we show that all the additional elements of $P$ (related to codes) can be simulated by a simulator which has only black-box access to $U$. See Appendix $\mathrm{C}$ for more details.

\subsection{Cast-as-intended Verifiability}

Cast-as-intended verifiability means that an honest voter can, with high probability, make sure that the ballot cast on her behalf by her voting platform and recorded in the ballot box by the voting server contains her intended choice. Our scheme provides cast-as-intended verifiability under one of the following cases: (1) The voter client is honest. (2) The following trust assumptions are satisfied:

V1. At most $t-1$ tellers are corrupted.

V2. The printing facility and the ballot sheet delivery channel are not corrupted.

The first case is trivial (note that the very purpose of code voting is to provide cast-as-intended verifiability in the case the voter client is not honest). We only need to assume that the voting client has means to check that the cast ballot has been in fact added to the ballot box and that there is a mechanism preventing any party from removing or modifying this ballot (which is the case if we assume that the ballot box is an instance of a secure bulletin board).

In the following we analyse the second case. Our result is as follows.

Theorem 3. Under the assumption V1 and V2, for any given honest voter (possibly using a dishonest voting platform) and for any of the $k$ voting options, the probability that the voter obtains the expected code (that is the code printed next to voter's choices), while the recorded ballot contains different choices for this voting option is not bigger than $\frac{1}{m-n-n^{\prime}}$ (plus a negligible value), where $m$ is the number of generated codes, $n$ is the total number of voters, and $n^{\prime}<n$ is the number of corrupted voters.

The proof of this result, similarly to the privacy result, does not depend on the details of the authentication mechanism nor on the details of the tallying phase.

The intuition behind this statement is that everything that the adversary can learn about code distribution is, essentially (up to cases of a negligible probability), what is explicitly given to him, that is (a) $n$ codes that have been decrypted by the tellers (b) $n^{\prime}$ remaining codes of dishonest voters (because the adversary gets to see all the codes of these voters). So, if the adversary wants to come up with the code corresponding to the opposite choice (for the considered voting option) of the honest voter in order to fool her, the best he can do is pick one of the remaining $m-n-n^{\prime}$ codes at random.

In order to prove this statement we, similarly to the privacy proof, use the ideal (honest) code generation procedure and replace PETs by the appropriate ideal functionality. In this setting the following is true. 
Because the code table is correct (correctly corresponds to the printed ballot sheets) and the results of PETs is correct too (as we are using the ideal functionality), it follows that the decrypted codes correspond to the actual voting options in the encrypted ballots. We can then show that, if the adversary had a strategy of guessing an unencrypted code of an honest voter with better probability than given by the blind guess as described above (where the adversary picks one of the possible codes at random), this would break the IND-CPA property of the underlying encryption scheme.

\section{References}

1. Ben Adida. Helios: Web-based Open-Audit Voting. In Paul C. van Oorschot, editor, Proceedings of the 17th USENIX Security Symposium, pages 335-348. USENIX Association, 2008.

2. Jordi Puiggalí Allepuz and Sandra Guasch Castelló. Internet voting system with cast as intended verification. In E-Voting and Identity - Third International Conference, VoteID 2011, Tallinn, Estonia, September 28-30, 2011, Revised Selected Papers, pages 36-52, 2011.

3. Stephanie Bayer and Jens Groth. Efficient Zero-Knowledge Argument for Correctness of a Shuffle. In David Pointcheval and Thomas Johansson, editors, Advances in Cryptology - EUROCRYPT 2012 - 31st Annual International Conference on the Theory and Applications of Cryptographic Techniques, volume 7237 of Lecture Notes in Computer Science, pages 263-280. Springer, 2012.

4. Véronique Cortier, David Galindo, Stéphane Glondu, and Malika Izabachène. Election verifiability for helios under weaker trust assumptions. In Computer Security - ESORICS 2014 - 19th European Symposium on Research in Computer Security, Wroclaw, Poland, September 7-11, 2014. Proceedings, Part II, pages 327-344, 2014.

5. David Galindo, Sandra Guasch, and Jordi Puiggali. 2015 Neuchâtel's Cast-asIntended Verification Mechanism. In E-Voting and Identity - 5th International Conference, VoteID 2015, Bern, Switzerland, September 2-4, 2015, Proceedings, pages 3-18, 2015.

6. R. Gennaro, S. Jarecki, H. Krawczyk, and T. Rabin. Secure Distributed Key Generation for Discrete-Log Based Cryptosystems. J. Cryptology, 20(1):51-83, 2007.

7. Kristian Gjøsteen. The norwegian internet voting protocol. In E-Voting and Identity - Third International Conference, VoteID 2011, Tallinn, Estonia, September 28-30, 2011, Revised Selected Papers, pages 1-18, 2011.

8. Kristian Gjøsteen. The norwegian internet voting protocol. IACR Cryptology ePrint Archive, 2013:473, 2013.

9. Rolf Haenni, Reto E. Koenig, and Eric Dubuis. Cast-as-Intended Verification in Electronic Elections Based on Oblivious Transfer. In Electronic Voting - First International Joint Conference, E-Vote-ID 2016, Bregenz, Austria, October 18-21, 2016, Proceedings, pages 73-91, 2016.

10. Rolf Haenni, Reto E. Koenig, Philipp Locher, and Eric Dubuis. CHVote system specification. IACR Cryptology ePrint Archive, 2017, 2017. 
11. James Heather, Peter Y. A. Ryan, and Vanessa Teague. Pretty Good Democracy for More Expressive Voting Schemes. In Dimitris Gritzalis, Bart Preneel, and Marianthi Theoharidou, editors, Computer Security - ESORICS 2010, 15th European Symposium on Research in Computer Security, volume 6345 of Lecture Notes in Computer Science, pages 405-423. Springer, 2010.

12. M. Jakobsson and A. Juels. Mix and Match: Secure Function Evaluation via Ciphertexts (Extended Abstract). In Advances in cryptology-ASIACRYPT 2000, page 162. Springer Verlag, 2000.

13. Jordi Puigalli and Sandra Guasch. Cast-as-intended verification in norway. In 5th International Conference on Electronic Voting 2012, (EVOTE 2012), Co-organized by the Council of Europe, Gesellschaft für Informatik and E-Voting.CC, July 11-14, 2012, Castle Hofen, Bregenz, Austria, pages 49-63, 2012.

14. P.Y.A. Ryan and V. Teague. Pretty Good Democracy. In Proceedings of the 17th International Workshop on Security Protocols 2009, 2009.

15. Ida Sofie Gebhardt Stenerud and Christian Bull. When reality comes knocking norwegian experiences with verifiable electronic voting. In 5th International Conference on Electronic Voting 2012, (EVOTE 2012), Co-organized by the Council of Europe, Gesellschaft für Informatik and E-Voting.CC, July 11-14, 2012, Castle Hofen, Bregenz, Austria, pages 21-33, 2012.

\section{A Encoding for Codes}

In this section we describe some potential instantiations for the family $\delta_{k}(i \in$ $\{1, \ldots, k\})$ of functions from the set of codes to the group $G$ such that individual codes $c_{1}, \ldots, c_{k}$ can be efficiently extracted from a product $\delta_{1}\left(c_{1}\right) \cdots \delta_{k}\left(c_{k}\right)$.

A simple construction is to use the initial small prime numbers $p_{0}, p_{1}, \ldots$ which belong to the group $G$ to represent consecutive bits of the binary representation of codes, as described below. Let us consider codes of the size of $l$ bits.

For a code $c$ with the binary representation $b_{0}, \ldots, b_{l-1}$, the function $\delta_{k}$ will use $l$ primes, say, $p_{k l-l}, \ldots p_{k l-1}$ as follows:

$$
\delta_{k}(c)=\prod_{j \in\{0, \ldots, l-1\}, b_{j}=1} p_{k l-l+j} .
$$

That is, we take the product of those primes that corresponds to non-zero bits. Note that different $\delta_{k}$ use different primes. For the decoding (computing the inverse of $\delta_{k}$ ) one simply needs to factorize the resulting value into the used small primes, which can be done efficiently. Note that, since the considered primes are in $G$, the product (2) is in $G$ as well, provided that it is smaller than $p$. Moreover, because we want to multiply encrypted $\delta_{k}(c)$, we need to make sure that the product of such values does not grow beyond $p$ as well. This imposes a limit on how many codes can we represent in a single ciphertext. For instance, for the standard 3072-bit ElGamal group, using this encoding we can have about 300 (exactly 296) independent bits, which allows us to handle 30 disjoint voting 
questions with 2-Base32-character codes, or 15 voting questions with 4-Base32character codes. If a ballot sheet needs to contain more than this, additional ciphertexts would have to be used.

We can, however, relatively easy obtain denser encoding. If, for instance, instead of using one prime to represent one bit of a code, we use groups of 32 consecutive prime numbers to represent 5 bits of the code (where only one prime in the group is set), then the same group can fit around 1000 bits in one ciphertext. This gives 100 disjoint voting questions with 2-character codes or 50 questions with 4-character codes, a number corresponding to relatively complex ballots.

\section{B Attack on [9]}

In order to understand the attack presented below, it may be useful for the reader to first consult the original paper [9]. It is worth noting that this attack scenario does not undermine the underlying ( $k$ out of $n$ )-OT scheme. It only utilizes the fact that a dishonest receiver in this scheme can obtain up to $k$ (but not more) values even if it does not follow the protocol. We describe here an attack for the case with $n=k=2$.

The intended run of the protocol is as follows. For the voter's choice $s=$ $\left(s_{1}, s_{2}\right)$, the voting platform (VP) prepares an OT query

$$
\boldsymbol{a}=\left(a_{1}, a_{2}\right), \quad \text { where } a_{j}=\Gamma\left(s_{j}\right) \cdot y^{r_{j}},
$$

for random $r_{j}$, where $y$ is the public election key. It also computes $b=g^{r_{1}+r_{2}}$. Let $a$ denote the product of elements of $\boldsymbol{a}$ that is $a_{1} \cdot a_{2}$. Note that $c=(b, a)$ is an ElGamal ciphertext (which, although not explicitly sent, will be considered to be the ciphertext cast by the voter) encrypting the plaintext $p=\Gamma\left(s_{1}\right) \cdot \Gamma\left(s_{2}\right)$ with randomness $r=r_{1}+r_{2}$. The VP sends $\boldsymbol{a}$ and $b$ along with a ZKP of knowledge of $r$ and $p$.

From the OT response, the VP can now compute the codes for $s_{1}$ and $s_{2}$ which are shown to the voter who provides the confirmation code and the protocol goes on. Here are the details of how the codes are retrieved. The OT response contains:

$$
\begin{aligned}
& a_{1}^{\alpha}, a_{2}^{\alpha}, y^{\alpha}, \\
& c_{1} \oplus H\left(\Gamma\left(s_{1}\right)^{\alpha}\right), c_{2} \oplus H\left(\Gamma\left(s_{2}\right)^{\alpha}\right), \ldots
\end{aligned}
$$

for some random $\alpha$, where $c_{1}$ and $c_{2}$ are the codes corresponding to choices $s_{1}$ and $s_{2}$. Knowing $r_{1}$ and $r_{2}$, the VP can compute $\Gamma\left(s_{1}\right)^{\alpha}$ and $\Gamma\left(s_{2}\right)^{\alpha}$ and, in turn, the codes $c_{1}, c_{2}$.

The dishonest run goes, for example, like this: For the voter's choice $s=$ $\left(s_{1}, s_{2}\right)$ as before, the VP prepares the OT query

$$
\tilde{\boldsymbol{a}}=\left(a_{1}, \tilde{a}_{2}\right), \quad \text { where } \quad \tilde{a}_{2}=\Gamma\left(s_{1}\right)^{7} \cdot \Gamma\left(s_{2}\right) \cdot y^{r_{2}}
$$


and sends $\tilde{\boldsymbol{a}}$ along with $b$ and a ZKP of knowledge of $r$ and the plaintext $\tilde{p}$, which is now $\Gamma\left(s_{1}\right)^{8} \cdot \Gamma\left(s_{2}\right)$. Jumping ahead, this plaintext will be rejected as invalid, but only after (mixing) and final decryption, when there is no visible link between the decrypted ballot and the voter.

Nevertheless, from the OT response, the VT can easily compute the codes for $s_{1}$ and $s_{2}$ and make the protocol proceed as if the intended, valid ballot was cast. To see this, we can notice that, given the OT response, the VT can compute values $\Gamma\left(s_{1}\right)^{\alpha}$ and $\left(\Gamma\left(s_{1}\right)^{7} \cdot \Gamma\left(s_{2}\right)\right)^{\alpha}$, from which it is easy to compute $\Gamma\left(s_{2}\right)^{\alpha}$ and the same codes $c_{1}$ and $c_{2}$ as in the honest run. These codes are delivered to the voter who then continues the procedure.

A straightforward countermeasure for this attack would be adding appropriate zero-knowledge proofs of correctness of each $a_{j}$, which however adds a significant computational overhead (it works in time $O(k \cdot n)$ ).

\section{Proof of Theorem 2}

We will now sketch the proof of this theorem, under the simplifying assumption that the code generation procedure is honest, i.e. carried out by one honest party (which is part of $P$ ). This assumption is justified by Theorem 1.

First, we can observe that

$$
\begin{aligned}
\operatorname{Prob}\left[P_{0} \| A \mapsto 1\right]= & \operatorname{Prob}\left[P_{0} \| A \mapsto 1 \wedge \boldsymbol{b}=(0,0)\right]+\operatorname{Prob}\left[P_{0} \| A \mapsto 1 \wedge \boldsymbol{b}=(0,1)\right]+ \\
& \operatorname{Prob}\left[P_{0} \| A \mapsto 1 \wedge \boldsymbol{b}=(1,0)\right]+\operatorname{Prob}\left[P_{0} \| A \mapsto 1 \wedge \boldsymbol{b}=(1,1)\right]
\end{aligned}
$$

where $\boldsymbol{b}$ represent the pair of flip-bits of the honest voters, and similarly for $P_{1}$. We can show that $\operatorname{Prob}\left[P_{0} \| A \mapsto 1\right]=\operatorname{Prob}\left[P_{1} \| A \mapsto 1\right]$ by showing that

$$
\begin{aligned}
& \operatorname{Prob}\left[P_{0} \| A \mapsto 1 \wedge \boldsymbol{b}=(0,0)\right] \equiv_{\text {negl }} \operatorname{Prob}\left[P_{1} \| A \mapsto 1 \wedge \boldsymbol{b}=(1,1)\right] \\
& \operatorname{Prob}\left[P_{0} \| A \mapsto 1 \wedge \boldsymbol{b}=(1,0)\right] \equiv_{\text {negl }} \operatorname{Prob}\left[P_{1} \| A \mapsto 1 \wedge \boldsymbol{b}=(0,1)\right]
\end{aligned}
$$

and so on. We focus on the first equation; the remaining cases are similar.

In order to prove this equation, we construct a simulator $S$ such that

$$
\operatorname{Prob}\left[P_{0} \| A \mapsto 1 \wedge \boldsymbol{b}=(0,0)\right] \equiv_{\text {negl }} \operatorname{Prob}\left[U_{0}\|S\| A \mapsto 1 \wedge \boldsymbol{b}=(0,0)\right]
$$

and

$$
\operatorname{Prob}\left[P_{1} \| A \mapsto 1 \wedge \boldsymbol{b}=(1,1)\right] \equiv_{\text {negl }} \operatorname{Prob}\left[U_{1}\|S\| A \mapsto 1 \wedge \boldsymbol{b}=(1,1)\right]
$$

and, furthermore, $S$ does not use $\boldsymbol{b}$ in any way (except that it picks this value at random). This gives

$$
\begin{aligned}
& \operatorname{Prob}\left[P_{0} \| A \mapsto 1 \wedge \boldsymbol{b}=(0,0)\right] \equiv_{\text {negl }} \operatorname{Prob}\left[U_{0}\|S\| A \mapsto 1 \wedge \boldsymbol{b}=(0,0)\right] \\
& =\frac{1}{4} \cdot \operatorname{Prob}\left[U_{0}\|S\| A \mapsto 1\right] \equiv_{\text {negl }} \frac{1}{4} \cdot \operatorname{Prob}\left[U_{1}\|S\| A \mapsto 1\right] \\
& =\operatorname{Prob}\left[U_{1}\|S\| A \mapsto 1 \mid \boldsymbol{b}=(1,1)\right] \equiv_{\text {negl }} \operatorname{Prob}\left[P_{1} \| A \mapsto 1 \mid \boldsymbol{b}=(1,1)\right]
\end{aligned}
$$


Which completes the proof.

We construct $S$ in the following way. Recall that $S$ must simulate all the honest components of $P$ and during this simulation it can interact with $U$ (in the role of an adversary for $U$ ).

Setup. $S$ forwards all the messages of the adversary related to the underlying protocol directly to $U$ and vice versa. It simulates the honest tellers in the procedures added in $P$ (generation of additional keys). Finally, for code table and ballot sheets it simulates the process as prescribed by the protocol with the exception of the code table of the honest voters which are created as follows.

$S$ first triggers the honest voters (of $U$ ) to cast their ballots. Let $u_{0}$ and $u_{1}$ be the encrypted choices of these voters taken from these ballots. $S$ then randomly picks the flip bits for the honest voters but ignores their values (these values will not be used in the following). The code table entries of the honest voters, as computed by $S$, are respectively

$$
c,\left(u_{0}^{\prime}, d_{0}\right),\left(u_{1}^{\prime}, d_{0}^{\prime}\right) \quad \text { and } \quad c^{\prime},\left(u_{0}^{\prime \prime}, d_{1}\right),\left(u_{1}^{\prime \prime}, d_{1}^{\prime}\right)
$$

where $c, c^{\prime}, d_{0}, d_{0}^{\prime}, d_{1}, d_{1}^{\prime}$ are generated like in the original code generation procedure (where, in particular the codes are picked at random), $u_{0}^{\prime}, u_{0}^{\prime \prime}$ are (independent) re-encryptions of $u_{0}$ and, similarly $u_{1}^{\prime}, u_{1}^{\prime \prime}$ are (independent) re-encryptions of $u_{1}$.

This produces the code table with exactly the same distribution as the honest procedure (under the given condition restricting $\boldsymbol{b}$ ).

Honest voters. For the first honest voter $S$ uses the original ballot (containing $\left.u_{0}\right)$ as produced by the honest voter when it was triggered and adds to it the encryption of $\tilde{b}=0$. Similarly for the second honest voter, but now $\tilde{b}$ is set to 1 .

PETs. By the security properties of the PET protocol, $S$ can simulate its shares without knowing the secret key share, if only the result of PET known, which holds in our case: for the honest voters the result of PETs is simply 'true'; for the dishonest voters the simulator can run the extraction algorithm to extract the used plaintexts from the zero-knowledge proofs included in the ballots, which allows the simulator to determine the result of the PETs, as it knows the plaintexts in the code tables of dishonest ballots (the simulator generated these plaintexts in the setup phase).

Decryption of codes. $S$ knows and uses its secret key share.

Note that, indeed, in this simulation, $S$ does not make any use of the flip bits (except that it generates it). 